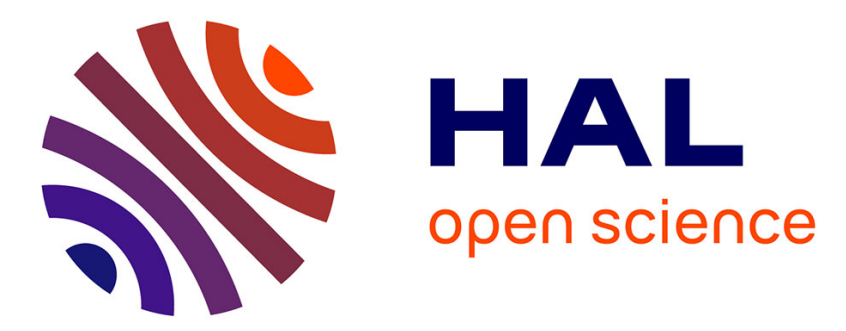

\title{
Repeated-Sprint Training in Hypoxia in International Rugby Union Players
}

\author{
Adam Beard, John Ashby, Ryan Chambers, Franck Brocherie, Grégoire P \\ Millet
}

\section{> To cite this version:}

Adam Beard, John Ashby, Ryan Chambers, Franck Brocherie, Grégoire P Millet. Repeated-Sprint Training in Hypoxia in International Rugby Union Players. International Journal of Sports Physiology and Performance, 2019, 14 (6), pp.850-854. 10.1123/ijspp.2018-0170 . hal-02544229

\section{HAL Id: hal-02544229 \\ https://hal-insep.archives-ouvertes.fr/hal-02544229}

Submitted on 16 Apr 2020

HAL is a multi-disciplinary open access archive for the deposit and dissemination of scientific research documents, whether they are published or not. The documents may come from teaching and research institutions in France or abroad, or from public or private research centers.
L'archive ouverte pluridisciplinaire HAL, est destinée au dépôt et à la diffusion de documents scientifiques de niveau recherche, publiés ou non, émanant des établissements d'enseignement et de recherche français ou étrangers, des laboratoires publics ou privés. 
Note. This article will be published in a forthcoming issue of the International Journal of Sports Physiology and Performance. The article appears here in its accepted, peer-reviewed form, as it was provided by the submitting author. It has not been copyedited, proofread, or formatted by the publisher.

Section: Brief Report

Article Title: Repeated-Sprint Training in Hypoxia in International Rugby Union Players

Authors: Adam Beard ${ }^{1}$, John Ashby ${ }^{2}$, Ryan Chambers ${ }^{2}$, Franck Brocherie ${ }^{3}$, and Grégoire P. Millet $^{1}$

Affiliations: ${ }^{1}$ ISSUL, Institute of Sport Sciences, Faculty of Biology and Medicine, University of Lausanne, Lausanne, Switzerland. ${ }^{2}$ Welsh Rugby Union, National Centre of Excellence, Vale of Glamorgan, Wales. ${ }^{3}$ Laboratory Sport, Expertise and Performance, Research Department, French Institute of Sport, Paris, France.

Journal: International Journal of Sports Physiology and Performance

Acceptance Date: November 18, 2018

(C)2018 Human Kinetics, Inc.

DOI: https://doi.org/10.1123/ijspp.2018-0170 
"Repeated-Sprint Training in Hypoxia in International Rugby Union Players" by Beard A et al.

International Journal of Sports Physiology and Performance

(c) 2018 Human Kinetics, Inc.

Title: Repeated-sprint training in hypoxia in international rugby union players

Submission type: Brief Report

Authors: Adam Beard ${ }^{1}$, John Ashby ${ }^{2}$, Ryan Chambers ${ }^{2}$, Franck Brocherie ${ }^{3}$, Grégoire P.

Millet $^{1}$

1. ISSUL, Institute of Sport Sciences, Faculty of Biology and Medicine, University of

Lausanne, Lausanne, Switzerland.

2. Welsh Rugby Union, National Centre of Excellence, Vale of Glamorgan, Wales.

3. Laboratory Sport, Expertise and Performance, Research Department, French Institute of Sport, Paris, France.

\title{
Corresponding Author
}

Adam Beard

Institute of Sport Sciences, Faculty of Biology and Medicine, University of Lausanne,

Lausanne, Switzerland.

Tel: +1.440 .732 .1938$

E-Mail: ajbeardo@hotmail.com

Preferred Running Head: RSH in international rugby union players

\author{
Abstract Word Count: 243 \\ Text-Only Word Count: 1988 \\ Tables: 1
}

Figures: 2 
"Repeated-Sprint Training in Hypoxia in International Rugby Union Players" by Beard A et al.

International Journal of Sports Physiology and Performance

(C) 2018 Human Kinetics, Inc.

\section{ABSTRACT}

Purpose: To investigate the effects of repeated-sprint training in hypoxia $v s$. in normoxia on World-level male Rugby Union players' repeated-sprint ability (RSA) during an international competition period. Methods: Nineteen players belonging to an international Rugby Union Senior male national team performed four sessions of cycling repeated-sprints (consisting of 3 $\times$ eight 10 -s sprints with 20 -s passive recovery) either in normobaric hypoxia (RSH, $3000 \mathrm{~m}$, $\mathrm{n}=10$ ) or in normoxia (RSN, $300 \mathrm{~m}$; $\mathrm{n}=9$ ) over a 2-wk period. At Pre- and Post-training intervention, RSA was evaluated using a cycling repeated-sprint test (6 x 10-s maximal sprint and 20-s passive recovery) performed in normoxia. Results: Significant interaction effects (all $\left.\mathrm{P}<0.05, \eta^{2}>0.37\right)$ between condition and time were found for RSA-related parameters. Compared to Pre-, maximal power significantly improved at Post- in RSH (12.84 \pm 0.83 vs. $13.63 \pm 1.03$ W.kg-1, $\left.\mathrm{P}<0.01, \eta^{2}=0.15\right)$ but not in RSN (13.17 \pm 0.89 vs. $13.00 \pm 1.01$ W.kg-1, $\left.\mathrm{P}=0.45,, \eta^{2}=0.01\right)$. Mean power was also significantly enhanced from Pre- to Post-intervention in $\mathrm{RSH}\left(11.15 \pm 0.58\right.$ vs. $\left.11.86 \pm 0.63 \mathrm{~W} . \mathrm{kg}-1, \mathrm{P}<0.001, \eta^{2}=0.26\right)$, while it remained unchanged in $\operatorname{RSN}\left(11.54 \pm 0.61\right.$ vs. $\left.11.75 \pm 0.65 \mathrm{~W} . \mathrm{kg}-1, \mathrm{P}=0.23, \eta^{2}=0.03\right)$. Conclusion: As little as four dedicated specific RSH sessions were beneficial to enhance repeated power production in World-level Rugby Union players. Although the improvement from RSA to game behaviour remains unclear, this finding appears of practical relevance since only a short preparation window is available prior to international Rugby Union games.

Keywords: Repeated-sprint training in hypoxia; Repeated-sprint ability; Team sports; Competition; Lower limbs; Rugby Union. 
"Repeated-Sprint Training in Hypoxia in International Rugby Union Players" by Beard A et al. International Journal of Sports Physiology and Performance

(C) 2018 Human Kinetics, Inc.

\section{Introduction}

Rugby Union is an intermittent sport often having periods of high-intensity efforts with incomplete recoveries ${ }^{1}$. Total distances reported in the literature have ranged between $4800-$ $5200 \mathrm{~m}$ for forwards and 5600-6000 $\mathrm{m}$ for backs, ${ }^{2}$ while sprint distances have ranged between $350-510 \mathrm{~m}^{2}$. Further, it has been demonstrated in other similar team sports such as Rugby League that as the level of competition rises from non-professional to professional level, the requirement for a higher number of sprints and need for higher strength and power abilities becomes paramount to game performance ${ }^{3}$. Rugby Union's international competitions tend to have small (i.e., 1-2 weeks) transition periods from national clubs to international games, this can pose problems for coaches and strength and conditioning experts in preparing players adequately. The search for useful methods able to shorten adaptation times within these small preparation periods from different levels of competition could provide a potential edge for physical performance. It was recently found that RSA performance was improved after a 2week intensified training period with elite soccer players after competition ${ }^{4}$. This may have good carryover to Rugby Union, which has a high number of repeated-sprint requirements ${ }^{2}$.

While RSA training is well accepted to improve this quality, ${ }^{5}$ utilizing RSA in hypoxic conditions (the so-called "repeated-sprint training in hypoxia", RSH) has shown superior results when comparing to similar training in normoxia $(\mathrm{RSN})^{6}$ in several sports ${ }^{7-10}$. It should be noted that while the majority of studies have shown positive RSA results when comparing $\mathrm{RSH}$ to $\mathrm{RSN},{ }^{6}$ there is still some debate on the overall effectiveness of RSH as well as debate on the mechanisms for the performance increase ${ }^{11,12}$. It has been speculated that the main underlying mechanism relies on the peripheral compensatory vasodilation in response to the hypoxia-induced hypoxemia, inducing a better resistance to fatigue in fast-twitch fibres, hence increasing the ability to repeat short bouts of exercise at maximal intensity ${ }^{13}$. One cannot rule 
"Repeated-Sprint Training in Hypoxia in International Rugby Union Players" by Beard A et al.

International Journal of Sports Physiology and Performance

(C) 2018 Human Kinetics, Inc.

out that RSH has also a positive impact on anaerobic and glycolytic pathway as shown previously ${ }^{14}$.

To date, all RSH studies have been conducted out of competition. Therefore, the aim of this study was assess the effectiveness of a few RSH sessions in the context on "real" highlevel competition schedules. For that, we evaluated the feasibility and effectiveness of an "inseason" RSH-shock microcycle (i.e., 4 additional sessions over a 2-wk period). We hypothesized that RSH would positively influence RSA performance in team-sport athletes during the short-term preparation to a game. Our main aim was to assess the effectiveness of few RSH sessions in the context on "real" high-level competition schedules.

\section{Methods}

\section{Participants}

Nineteen World-level players $(187.0 \pm 5.5 \mathrm{~cm}$ and $103.8 \pm 11.6 \mathrm{~kg})$ belonging to an international Rugby Union Senior male national team participated in the study as part of their normal national squad training schedule in preparation for the Six Nations tournament. All players were members of the same team and followed the same training/recovery program. They were followed by the same nutritionist who provided similar allocated/supplied meals and supplementation. While respecting the recommendation of the Declaration of Helsinki, ethics committee clearance was not required as training/testing data collection arise as a condition of players' duties ${ }^{15}$.

\section{Experimental design}

The study consisted of two testing sessions before (Pre-) and after (Post-) a 2-wk "inseason" training period including 4 RSH/RSN sessions. Players were matched regarding their normal units training groups, backs $(23.0 \pm 1.9 \mathrm{yr} ; 186.4 \pm 6.6 \mathrm{~cm} ; 93.7 \pm 8.6 \mathrm{~kg})$ and forwards $(25.6 \pm 3.7 \mathrm{yr} ; 189.4 \pm 4.5 \mathrm{~cm} ; 112.9 \pm 4.9 \mathrm{~kg})$ and were randomized in either normobaric hypoxia (RSH, 3000 m, $\mathrm{F}_{\mathrm{I}} \mathrm{O}_{2}, 13.8 \%$; $\left.=10\right)$ or normoxia (RSN, $300 \mathrm{~m} ; \mathrm{F}_{\mathrm{I}} \mathrm{O}_{2}, 20.9 \%$; $=9$ ). 
"Repeated-Sprint Training in Hypoxia in International Rugby Union Players" by Beard A et al.

International Journal of Sports Physiology and Performance

(C) 2018 Human Kinetics, Inc.

The 2-wk period consisted of 2 small sided games sessions, 8 specific Rugby sessions, 2 captains run sessions, 4 weight training sessions and 4 recovery sessions. This training intervention was inserted in the 'normal' planned program of the team. No additional tapering period was added.

\section{Specific repeated-sprint training}

In addition to their usual national squad training, in a double blinded fashion players performed four ergocycle-based (Wattbike Ltd, Nottingham, UK) RSH/RSN sessions over a 2-wk period. Wattbike repeated sprint training was a common practice in the team (as in many Rugby teams worldwide). Therefore the players did not require familiarization to produce maximal effort during the 10-s sprints. Two sessions per week were completed in a normobaric hypoxic chamber (POWERbreathe, Southam, England, UK) which consisted of three sets of eight 10 -s sprints with 20 -s intervals of passive recovery and 2 min between each set, players were given their maximum power output scores as motivation and were encouraged to complete all repetitions maximally while trying to produce the highest power output possible. Overview of the training sessions consisted of a 5-min warm-up at 80-90 rpm, followed by a subsequent 1-min block with a 10-s submaximal sprint and 50-s passive recovery and a second 1-min block repeating block 1 but with greater emphasis on maximal sprint. After 1 min of rest, the training exercise was initiated. Players were given a count down and encouraged energetically to complete every sprint maximally. Total hypoxic exposure was 24 min for RSH while cool-down was completed in normoxic conditions for both RSH and RSN groups. The ergo cycles were calibrated according to the manufacturer guideline prior to each training session.

\section{Repeated-sprint ability test}

RSA was evaluated using an electronic braked cycling ergometer (Lode Excalibur Sport; Lode Medical Technology, Groningen, The Netherlands) set at a fixed torque of 0.8 
"Repeated-Sprint Training in Hypoxia in International Rugby Union Players" by Beard A et al.

International Journal of Sports Physiology and Performance

(C) 2018 Human Kinetics, Inc.

$\mathrm{Nm} \cdot \mathrm{kg}^{-1}$ and consisted of six repeated-cycling 10-s maximal sprints with $20 \mathrm{~s}$ of passive recovery performed in normoxia. RSA-related parameters such as peak power output (PPO) and mean power output (MPO; calculated over the entire 10-s effort) were recorded for each of the six repetitions. PPO were used to calculate the sprint decrement score (\%) as follows: [1 - (total power/ideal power) $\times 100$; where total power is the cumulated PPO across repetitions, while ideal power refers to the highest PPO during one single 10-s sprint multiplied by the number of repetitions 5 .

\section{Statistical analysis}

Data are presented as mean $( \pm \mathrm{SD})$ or relative changes $(\%)$ unless otherwise stated. Normal distribution of the data was tested using the Shapiro-Wilk test. Two-way repeatedmeasures analysis of variance (ANOVA) with 1 between factor (condition; RSH vs. RSN) and 1 within factor (time; Pre- vs. Post-) were used to compare RSA-related performance variables. Multiple comparisons were made using Holm-Sidak post hoc test. For each ANOVA, partial eta-squared $\left(\eta^{2}\right)$ was calculated as measure of effect size. According to Cohen ${ }^{16}$, values of 0.01 , 0.06 and 0.14 were considered as small, medium and large, respectively. All analyses were made using Sigmaplot 11.0 software (Systat Software, Inc., San Jose, CA, USA). Null hypothesis was rejected at $\mathrm{P}<0.05$.

\section{Results}

\section{Training exposure}

Players using $\mathrm{RSH}$ were exposed to 24 min of normobaric hypoxia (3000 m, $\mathrm{F}_{\mathrm{I}} \mathrm{O}_{2}$, $13.8 \%$ ) per training session, thereby totalizing 96 min over the entire intervention. Conversely, RSN spend 24 min in normoxia per training session for a total of 96 min. 
"Repeated-Sprint Training in Hypoxia in International Rugby Union Players" by Beard A et al.

International Journal of Sports Physiology and Performance

(C) 2018 Human Kinetics, Inc.

\section{Repeated-sprint ability}

Significant effects $\left(\mathrm{P}<0.05, \eta^{2}>0.37\right)$ between condition and time were found for RSArelated parameters. In $\mathrm{RSH}$, the $10-\mathrm{s} \mathrm{PPO}$ were significantly better $(\mathrm{P}<0.05)$ for all repetitions, except the fifth sprint (Figure 1). No significant change was observable in RSN (Figure 1). Compared to Pre-, PPO significantly improved at Post- in RSH $(12.84 \pm 0.83$ vs. $13.63 \pm 1.03$ $\left.\mathrm{W}, \mathrm{P}<0.01, \eta^{2}=0.15\right)$ but not in $\mathrm{RSN}\left(13.17 \pm 0.89\right.$ vs. $\left.13.00 \pm 1.01 \mathrm{~W}, \mathrm{P}=0.45, \eta^{2}=0.01\right)$ (Figure 2). MPO was also significantly enhanced from Pre- to Post-intervention in RSH (11.15 \pm 0.58 vs. $\left.11.86 \pm 0.63 \mathrm{~W}, \mathrm{P}<0.001, \eta^{2}=0.26\right)$, while it remained unchanged in $\mathrm{RSN}(11.54 \pm 0.61$ vs. $11.75 \pm 0.65 \mathrm{~W} ; \mathrm{P}=0.23, \eta^{2}=0.03$ ) (Figure 2). Sprint decrement did not change between conditions (pooled data: $-12.6 \pm 3.7 \%$ at Pre- vs. $-11.2 \pm 3.4 \%$ at Post-; $P=0.12, \eta^{2}=0.08$ ) throughout the protocol.

\section{Discussion}

The present study investigated the effect of RSH vs. RSN on RSA-related parameters in World-level Rugby Union players during a preparation phase preceding an international competition. The main findings were that four RSH sessions over a 2-wk shock microcycle preparation period resulted in greater improvements (i.e., higher lower-limb repeated power output) than similar training in normoxia (RSN). Since players were able to view their power outputs during all training sessions, one cannot rule out an influence of motivational factors. It also guarantees that sessions were performed at maximal intensity in these very competitive subjects. Concerning RSA reproducibility, Watt et al. ${ }^{17}$ reported standard errors of measurement for peak and mean power of 1.6 and $1.2 \%$, respectively, indicating adequate precision. Furthermore, Wattbike cycle ergometer proved acceptable accuracy (2.6\%) when compared to SRM (1.1\%) considered as the gold standard in cycling measurements ${ }^{18}$. 
"Repeated-Sprint Training in Hypoxia in International Rugby Union Players" by Beard A et al. International Journal of Sports Physiology and Performance

(C) 2018 Human Kinetics, Inc.

The number of sessions completed in recent $\mathrm{RSH}$ research has ranged between six ${ }^{7,8}$ and fifteen sessions ${ }^{19}$ with the average number being $9 \pm 3$ from Brocherie's meta-analysis on $\mathrm{RSH}^{6}$. Galvin et al. ${ }^{9}$ used $12 \mathrm{RSH}$ sessions with academy level Rugby Union players and showed a two-fold greater improvement in RSH vs. RSN. Further, Hamlin et al. ${ }^{10}$ recently reported anaerobic, but not aerobic, performance changes after only $6 \mathrm{RSH}$ sessions completed over 3 weeks in well-trained Rugby Union players. This appears consistent to our results, which indicates that World-level Rugby Union players improved RSA-related PPO and MPO with only $4 \mathrm{RSH}$ sessions. In this study the international players produced on average around 13 W.kg-1. Hamlin et al. (2017) reported Peak Power figures in well trained Rugby players of between 9.98 and $10.8 \mathrm{~W} . \mathrm{kg}-1$. Faiss et al. ${ }^{13}$ reported a significant increase in the number of sprints in RSH $(67 \pm 11 \%$ vs. $69 \pm 8 \%$ from Pre- to Post-, p<0.01) vs. RSN $(65 \pm 7 \%$ vs. $68 \pm$ $9 \%, \mathrm{NS})$ with no differences in MPO improvement (+6 $\pm 7 \%$ vs. $+7 \pm 8 \%)$ following 8 cyclingbased (192 min) RSH or RSN sessions. While our study applied a similar testing and training protocol to Faiss et al., ${ }^{13}$ training timelines (4 vs. 8), type of sport (Rugby Union vs. cycling), practice level (World-level vs. moderately-trained participants) as well as anthropometrical differences may have accounted for dissimilar results. Further, due to the sport-specific requirements (i.e., intermittent- vs. endurance-based activities), it is reasonable to postulate that the World-level Rugby Union players investigated here would have a greater fast-twitch fibres proportion than moderately-trained cyclists, however caution should be taken regarding interpretation as most powerful players (likely having higher type-II fiber composition) may have benefit to a greater extent from RSH. One may also argue that the wattbike training is not specific actions on the field. However Hamlin et al. (2017) showed that power output during training on a cycle ergometer increased throughout the RSH period which corresponded to an eventual enhancement in over-ground repeat running sprint performance. 
"Repeated-Sprint Training in Hypoxia in International Rugby Union Players" by Beard A et al.

International Journal of Sports Physiology and Performance

(C) 2018 Human Kinetics, Inc.

It is known that training load during this international 2-week preparation period has certainly been influenced by player position given the part of specific positional training in Rugby Union ${ }^{20}$ however as described in the methods the players all follow a similar overview periodization (The 2-wk period consisted of 2 small sided games sessions, 8 specific Rugby sessions, 2 captains run sessions, 4 weight training sessions and 4 recovery sessions)

\section{Conclusion}

As little as four dedicated specific RSH sessions were beneficial to enhance repeated power production in World-level Rugby Union players. Although the improvement from RSA to game behaviour remains unclear, this finding appears of practical relevance since only a short preparation window is available prior to international Rugby Union games.

\section{Practical applications}

This study would provide coaches and strength and conditioning experts useful guidelines on how to apply RSH in a very short adaptation window. Further investigations are warranted to deeper understand the RSH periodization during the various cycle of the teamsport season, including the international period. In addition, utilizing an upper-body dominant exercise regimen such as the one employed by Faiss et $a l,{ }^{8}$ in cross-country skiing could be also beneficial in some sports - such as the rugby-related (i.e., union, league, AFL), field and ice hockey, lacrosse and combat sports - that require high repeated-sprint/power abilities in both lower and upper limbs.

\section{Acknowledgements}

The authors acknowledge the national team players who participated and the support staff for their help. 
"Repeated-Sprint Training in Hypoxia in International Rugby Union Players" by Beard A et al.

International Journal of Sports Physiology and Performance

(C) 2018 Human Kinetics, Inc.

\section{References}

1. Duthie G, Pyne D, Hooper S. Applied physiology and game analysis of rugby union. Sports Med. 2003;33(13):973-991.

2. Quarrie KL, Hopkins WG, Anthony MJ, Gill ND. Positional demands of international rugby union: evaluation of player actions and movements. J Sci Med Sport. 2013;16(4):353-359.

3. Gabbett TJ. Influence of playing standard on the physical demands of professional rugby league. J Sports Sci. 2013;31(10):1125-1138.

4. Christensen PM, Krustrup P, Gunnarsson TP, Kiilerich K, Nybo L, Bangsbo J. VO2 kinetics and performance in soccer players after intense training and inactivity. Med Sci Sports Exerc. 2011;43(9):1716-1724.

5. Girard O, Mendez-Villanueva A, Bishop D. Repeated-sprint ability - part I: factors contributing to fatigue. Sports Med. 2011;41(8):673-694.

6. Brocherie F, Girard O, Faiss R, Millet GP. Effects of Repeated-Sprint Training in Hypoxia on Sea-Level Performance: A Meta-Analysis. Sports Med. 2017;47(8):16511660.

7. Brocherie F, Girard O, Faiss R, Millet GP. High-intensity intermittent training in hypoxia: a double-blinded, placebo-controlled field study in youth football players. $J$ Strength Cond Res. 2015;29(1):226-237.

8. Faiss R, Willis S, Born DP, et al. Repeated double-poling sprint training in hypoxia by competitive cross-country skiers. Med Sci Sports Exerc. 2015;47(4):809-817.

9. Galvin HM, Cooke K, Sumners DP, Mileva KN, Bowtell JL. Repeated sprint training in normobaric hypoxia. Br J Sports Med. 2013;47 Suppl 1:i74-79.

10. Hamlin MJ, Olsen PD, Marshall HC, Lizamore CA, Elliot CA. Hypoxic Repeat Sprint Training Improves Rugby Player's Repeated Sprint but Not Endurance Performance. Front Physiol. 2017;8:24.

11. Inness MW, Billaut F, Aughey RJ. Team-Sport Athletes' Improvement of Performance on the Yo-Yo Intermittent Recovery Test Level 2, but Not of Time-Trial Performance, With Intermittent Hypoxic Training. Int J Sports Physiol Perform. 2016;11(1):15-21.

12. Montero D, Lundby C. Effects of Exercise Training in Hypoxia Versus Normoxia on Vascular Health. Sports Med. 2016;46(11):1725-1736.

13. Faiss R, Leger B, Vesin JM, et al. Significant molecular and systemic adaptations after repeated sprint training in hypoxia. PLoS One. 2013;8(2):e56522.

14. Puype J, Van Proeyen K, Raymackers JM, Deldicque L, Hespel P. Sprint interval training in hypoxia stimulates glycolytic enzyme activity. Med Sci Sports Exerc. 2013;45(11):2166-2174.

15. Winter EM, Maughan RJ. Requirements for ethics approvals. J Sports Sci. 2009;27(10):985. 
"Repeated-Sprint Training in Hypoxia in International Rugby Union Players" by Beard A et al.

International Journal of Sports Physiology and Performance

(c) 2018 Human Kinetics, Inc.

16. Cohen JC. Statistical Power Analysis for the Behavioral Sciences. 1988.

17. Watt KK, Hopkins WG, Snow RJ. Reliability of performance in repeated sprint cycling tests. J Sci Med Sport. 2002;5(4):354-361.

18. Hopker J, Myers S, Jobson SA, Bruce W, Passfield L. Validity and reliability of the Wattbike cycle ergometer. Int J Sports Med. 2010;31(10):731-736.

19. Goods PS, Dawson B, Landers GJ, Gore CJ, Peeling P. No Additional Benefit of Repeat-Sprint Training in Hypoxia than in Normoxia on Sea-Level Repeat-Sprint Ability. J Sports Sci Med. 2015;14(3):681-688.

20. Lacome M, Piscione J, Hager JP, Bourdin M. A new approach to quantifying physical demand in rugby union. J Sports Sci. 2014;32(3):290-300. 
"Repeated-Sprint Training in Hypoxia in International Rugby Union Players" by Beard A et al. International Journal of Sports Physiology and Performance

(C) 2018 Human Kinetics, Inc.
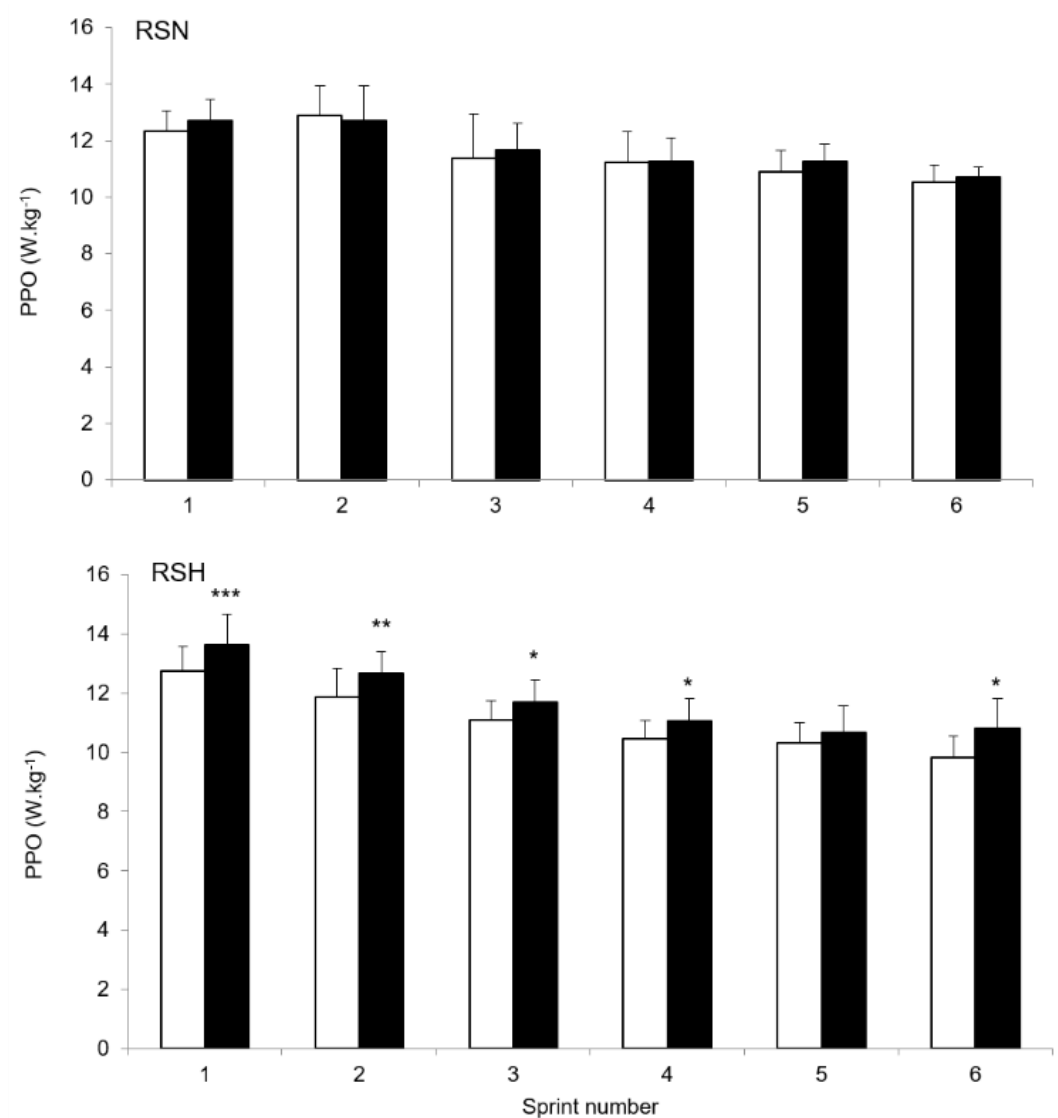

Figure 1. Peak power output (PPO) in successive sprints during the repeated-cycling sprint test before (Pre-) and after (Post-) specific repeated sprint training in hypoxia (RSH) or in normoxia $(\mathrm{RSN})$. Values are mean $\pm \mathrm{SD}$. Significant differences from Pre- test, $* \mathrm{P}<0.05$; and $* * *$ $\mathrm{P}<0.001$; 

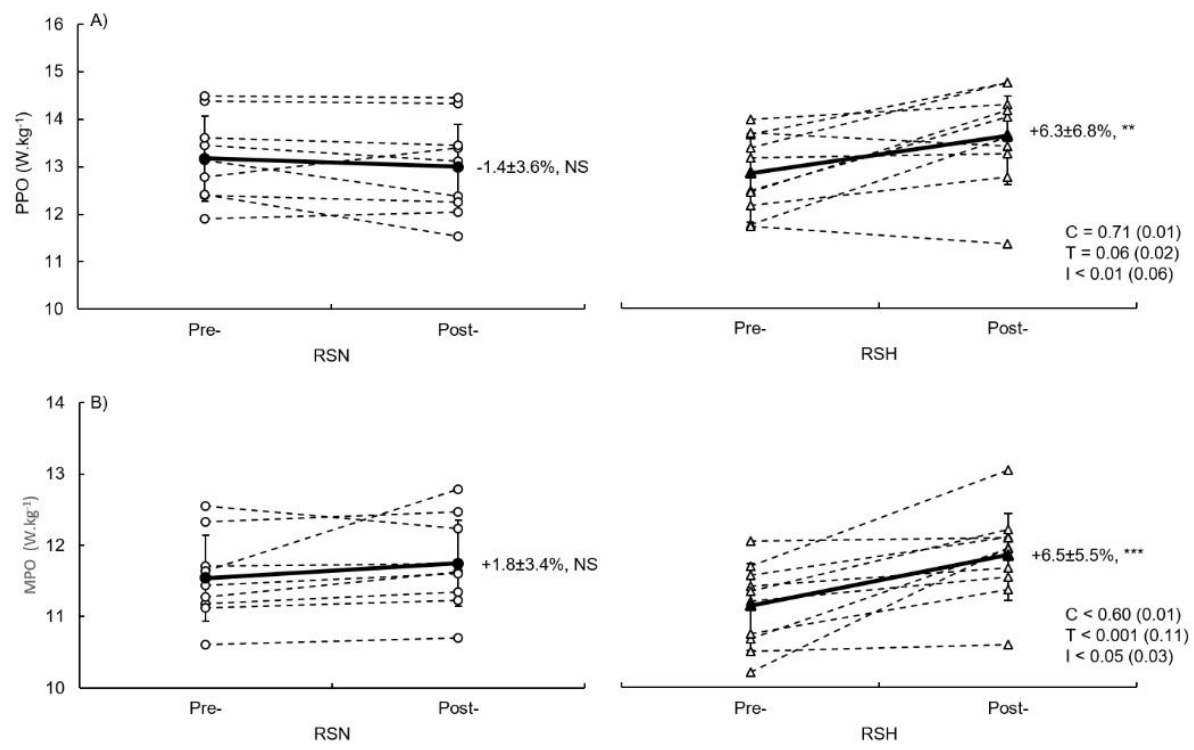

Figure 2. Individual (small symbols, dotted thin lines) and average (large symbols, dark line) in peak power output (PPO; A) and mean power output (MPO; B) before (Pre-) and after (Post) repeated-sprint training in hypoxia $(\mathrm{RSH} ; \mathbf{\Delta})$ or normoxia $(\mathrm{RSN} ; \circ)$. Values are mean $\pm \mathrm{SD}$. $C$, condition; T, time, and I, interaction. Significant differences from Pre- test, ${ }^{*} \mathrm{P}<0.05$; and $* * * \mathrm{P}<0.001$; significant differences between groups, \# $\mathrm{P}<0.05$. 
"Repeated-Sprint Training in Hypoxia in International Rugby Union Players" by Beard A et al. International Journal of Sports Physiology and Performance

(c) 2018 Human Kinetics, Inc.

Table 1. World-level Rugby Union players' characteristics in repeated-sprint training in hypoxia (RSH) and_repeated-sprint training in normoxia (RSN) group.

\begin{tabular}{lcc}
\hline & RSN $(\mathbf{N}=9)$ & RSH $(\mathbf{N}=10)$ \\
\hline Age $(\mathrm{y})$ & $23.0 \pm 1.9$ & $25.6 \pm 3.7$ \\
Height $(\mathrm{cm})$ & $186.4 \pm 6.6$ & $189.4 \pm 4.5$ \\
Body mass $(\mathrm{kg})$ & $93.7 \pm 8.6$ & $112.9 \pm 4.9 \# \# \#$ \\
International games $(\mathrm{Nb})$ & $40.0 \pm 27.7$ & $40.5 \pm 24.4$ \\
\hline
\end{tabular}

Significant differences between groups, \# P<0.001. 